\title{
Oxigenoterapia domiciliaria en niños con displasia broncopulmonar
}

\author{
M.C. Juan Pablo Beca I. ${ }^{1}$; M.C. Jorge Torres P. ${ }^{1}$; M.C. Juan Sfeir . $^{1}$; M.C. Germán Rodriguez $\mathrm{H}^{1}{ }^{1}$; \\ E.U. Miriam Peña A. '; Kigo. Alejandra Vija $I^{2}$
}

\section{Oxygen therapy at home for patients with bronchopulmonary dysplasia}

\begin{abstract}
Eight babies with severe bronchopulmonary dysplasia (BPD) were discharged from a private haspital at Santiago, Chile, on a program that provided oxygen therapy at home. Mean gestational age was $27 \pm 2$ weeks and mean birth weight was $1,181 \pm 353 \mathrm{~g}$. Discharge was decided af ter stabilization of arterial $\mathrm{PO}_{2}$ or $\mathrm{O}_{2} \mathrm{Sat}, \mathrm{PCO}_{2}<50 \mathrm{mmHg}$ and adequate weight gain. Prior to discharge parents were trained in $\mathrm{O}_{2}$ administration and cardiopulmonary resucitation. Oxygen was given at home for a mean of 71 days (range 7 to 339). There were no complications secondary to oxygen therapy. At follow up in only two cases additional hospitalizations wete needed, in both instances because of in tercurrent respiratory infections. Weight gain between $3^{\text {th }}$ and $50^{\text {th }}$ percentile were seen in all cases. Oxygen therapy at home appears to be a good alternative in the treatment of infants with BPD, and it reduces significantly hospital stay and costs. Both optimal family conditions and adequate support at home are needed.

(Key wordg: bronchopulmonary dysplasia, oxygen therapy at home, on a program for oxygen therapy at home.)
\end{abstract}

La displasia broncopulmonar (DBP) o enfer. medad pulmonar crónica es una afección propia de los recién nacidos de bajo peso, sometidos a ventilación mecánica, atribuida a múltiples factores; entre los que destacan la inmadurez pulmonar, el barotrauma, causado por los respiradores y el efecto tóxico del oxígeno en el pulmón. Su incidencia aumenta, a pesar de los múltiples esfuerzos de prevención, a medida que aumenta la sobrevida de reciến nacidos de menor peso $y$ menor edad gestacional ${ }^{1-3}$. Esta ha sido la experiencia tanto extranjera como

1. Servicio de Neonatologia, Clínica Alemana.

2. Kinesiólogo. nacional, en la cual, si bien ha habido escasas publicaciones $^{4-6}$, los casos han aumentado en numero en casi todas las unidades de neonatología en los últimos affos. Los niños más graves requieren largas hospitalizaciones, debidas a la prolongada dependencia de oxígeno, que puede durar varios meses, como a las frecuentes enfermedades intercurrentes.

Con el objeto de evaluar la factibilidad, ventajas y eventuaies complicaciones de la oxigenoterapia en domicilio como parte del tratamiento de la DBP, se estudió retrospectivamente un grupo de niños afectados que fueron dados de alta con oxigenoterapia en su domicilio con el fin de abreviar la hospitalización y favorecer la vida familiar. 


\section{MATERIAL Y METODO}

Se estudió en detalle la ficha clinica de hospitalización $y$ la ficha de control ambulatorio de 8 casos de DBP dados de atta con oxigenoterapia en su domicilio. Todos nacieron $y$ fueron tratados en el servicio de neonatología de la Clínica Alemana de Santiago entre los años 1983 y 1989 , período en el cual otros 11 casos fueron dados de alta sin oxízeno.

El diagnóstico de DBP se formuló de acuetdo a los criterios propuestos por Bancalari ${ }^{7}$ : RN de pretérmino tratados con ventilación mecárica en la primera semana de vida, signos respiratorios persistentes, dependencia de oxígeno por más de 28 días y radiología característica con zonas de opacidad e hipertransparencia alternando en ambos campos pulmonares.

E) tratamiento de la DHP incluyó ventilación mecánica, por plazos muy variados en los diversos casos; oxigenoterapia controlada; uso intermitente de furosemida; aminofilina oral; antibióticos cuando hubo sospecha fundada de infección bacteriana y apoyo nutricional. El alta de la clínica se decidió cuando se logró estabilización de la oxemia o de la saturación de hemoglobina, con oxigenoterapia suministrada mediante naricera y flujo menor de $1,5 \mathrm{l} \cdot \mathrm{min}$, retención de $\mathrm{CO}_{2}$ menor de $50 \mathrm{mmHg}$ y curva de peso en ascenso.

Antes del alta se adjestró a los padres en el cuidado de los niños, el uso y limpieza de los equipos de oxigenoterapia y de monitores, cuando éstos se emplearon, asi como en las técnicas básicas de reanimaclón. El equipo empleado en casa incluyó balones de oxigeno de $9 \mathrm{~m}^{3}$, un balón pequeño de transporte con sus respectivos flujómetros $y$ humidificadores, naticeras pediátricas y pipetas de aspiración. En dos casos se ussion motores de aspiración y en un paciente montitor de apnea.

Una vez en el domiculio se hizo control semanal por enfermera, kinesiterapia respiratoria dos veces por semana $y$ control pediátrico, al comienzo semanal, luego quincenal. Los prineros casos se controlaron con gases en sangre arterial, tomados en domicilio y el $\mathrm{O}_{2}$ sc suspendió en base a la observación clínica. Los últimos 4 casos se hospitalizaron por un día para controlar la tensión transcutánea de ox ígeno y la saturación de hemoglobina, gases arteriales y radiografía de tốrax antes de suspender el ox ígeno.

El seguimiento se realizó en los respectivos domr cilios y en la consulta externa, con interconsultas a especialistas cuando fue necesario. El progreso ponderal se registró empleando las curvas normales del Centro Nacional de Estadistica de Salud de EUA (NCHS) ${ }^{8}$.

\section{RESULTADOS}

En el periodo analizado hubo 19 casos de DBP, todos estaban vivos al alta y en 8 se indicó oxígeno en el domicilio, de acuerdo al criterio previamente descrito. La edad gestacional promedio fue $27 \pm 2$ semanas (rango 24 a 30 ) y el peso de nacimiento promedio fue $1.181 \pm 353 \mathrm{~g}$ (rango 700 a 1.970). La duración de la ventila. ción mecánica fue de 5 a 225 días y la hospitalización de 57 a 284 días. La oxigenoterapia intrahospitalaria duró lo mismo que la hospitalización, en promedio, 127 días (rango 57 a 284 días). La oxigenoterapia en domicilio duró, en promedio, $71 \mathrm{DE} 102$ días (rango 7 a 339 días). Hubo excelente aceptación familiar de la técnica y no se presentaron dificuitades ni complicaciones con su uso.

El seguimiento clínico de los pacientes se ha prolongado por 5,5 años en el primer caso, y por 3 meses en el último. La morbilidad general después del alta hospitalaria se resume en la tabla. Dos pacientes requirieron rehospitalización, debido a bronconeumonía y 3 de los 8 evolucionaron con sindrome bronquial obstructivo recidivante. El incremento ponderal, calculado para la edad postnatal corregida, se mantuvo entre los percentiles 3 y 50 de la curva de crecimiento normal del NCHS (figura).

Tabla

Morbilidad después de alta

\begin{tabular}{llll}
\hline Caso & Seguimiento & \multicolumn{1}{c}{ Morbilidad } \\
\hline 1 & $5 \frac{1}{2}$ & a & Obstrucción laríngea, hipoacusia, estrabismo, retardo lenguaje. \\
2 & 1 & a & Vomitador, SBOR, retardo psicomotor leve. \\
3 & $1 / \frac{1}{2}$ & a & Bronconeumonía, IPPV, SBOR. \\
4 & 1 & a & Hidrocefalia, espasticidad, miop í. \\
5 & 3 & a & Retardo lenguaje. \\
6 & $3 \frac{1}{2}$ & a & Bronconeumonía, SBOR, retardo lenguaje. \\
7 & $5 \frac{1}{2}$ & $\mathrm{~m}$ & Ditatación ventricular leve. \\
8 & 3 & m & Sin enfermedides agregadas. \\
\hline
\end{tabular}

SBOR : Síndrome bronquial obstructivo recidrante.

IPPV : Ventilación con presión positiva intermitente. 


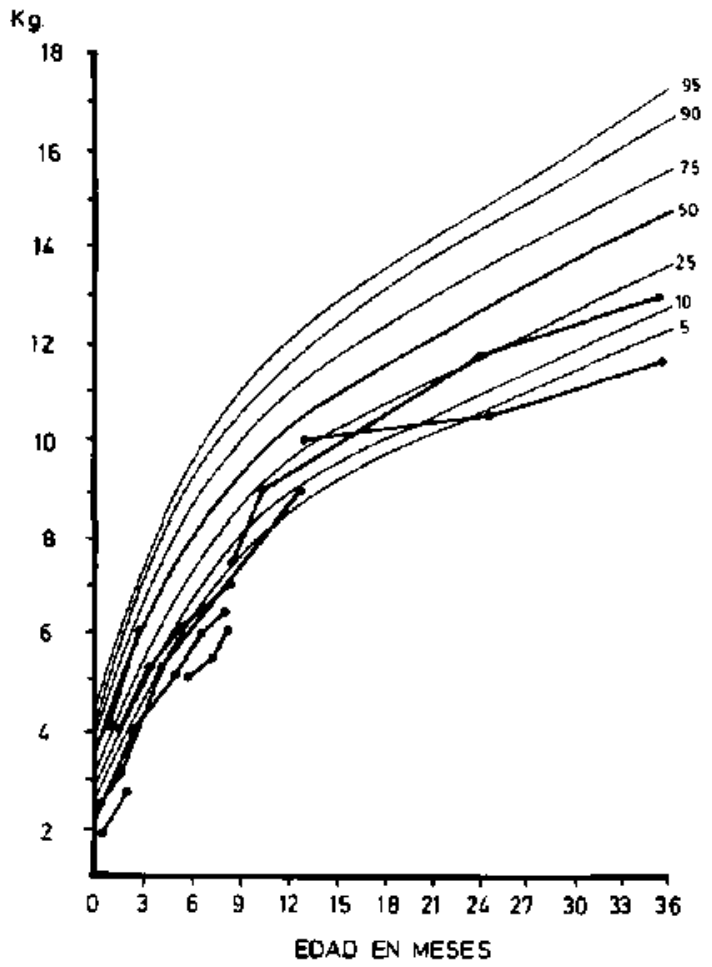

Figura: Evolución del peso en 8 pacientes con DBP 亿ratados con oxigenoterapia en domicilio. Valores normales de las cartas del NCHS ${ }^{\mathrm{B}}$.

\section{DISCUSION}

La elevada incidencia de displasia broncopulmonar entre los recién nacidos de muy bajo peso que sobteviven no ha podido ser reducida a pesar de los numerosos intentos de prevención ${ }^{1-3,9-11}$, causando hospitalizaciones prolongadas de alto costo, morbilidad asociada y alteraciones de las relaciones familiares. Con el objeto de evitar estos problemas se ha empleado desde 1976 oxigenoterapia controlada en domicilio en casos debidamente seleccionados ${ }^{i 2-14}$.

Las características clínicas de edad gestacional, peso al nacer, tiempo en ventilación mecánica y enfermedades asociadas, así como el criterio de selección de pacientes para tratamiento con oxígeno en los domicilios de esta serie, son similares a los empleados por otros autore ${ }^{12-16}$. Es importante destacar, entre las condiciones necesarias, la estabilidad físiológica del paciente $y$ sus demandas y la adecuada preparación y adiestramiento de los padres. La determinación del requerimiento de oxígeno antes del alta exige vigilar a los niños con mediciones de saturación o tensión transcutánea de oxígeno en diversas condiciones como sueño, alimentación y activi$\operatorname{dad}^{12,17}$. Igual método se recomienda para decidir la suspensión de oxígeno, la cual debe hacerse cautelosamente, ya que estos pacientes tienen elevados consumos de oxígeno ${ }^{18-20}$ y la suspensión prematura del aporte adicional se traduce en detención de crecimiento y mayor hipertensión pulmonar ${ }^{16,21}$. En esta experiencia, los cuatro últimos casos fueron hospitalizados para decidir la suspensión del tratamiento, según los resultados de la saturación de oxígeno y gases arteriales.

El equipo empleado para administrar oxígeno en domicilio fue mínimo. Sin embargo, es reco. mendable emplear monitores de apnea o cardiorrespiratorios en el domicilio, ya que los pacientes con DBP tienen 7 veces más riesgo de muerte súbita que sujetos control ${ }^{22}$. En esta serie se empleó monitor de apnea en un solo caso y no ocurrieron situaciones de apnea grave o de amenaza frustra de muerte súbita. Los costos de la oxigenoterapia en domicilio no se determinaron, ya que fueron cubiertos, con apoyos previsionales variados, por cada familia. Sin embargo, se ha demostrado que son menores que los de hospitalización ${ }^{13}, 23$.

El crecimiento de los pacientes durante el periodo en el cual recibieron oxigeno en domicilio, si bien no se pudo comparar a un grupo control, fue progresivo y lineal entre los percentiles 3 y 50, en contraste con la detención de crecimiento que ocurre si se suspende temporalmente el oxígeno en pacientes con $\mathrm{DBP}^{16}$, constituyéndose la ganancia ponderal en un signo indirecto de adecuada oxigenación.

La buena aceptación, por la familia, de la oxigenoterapia en domicilio, sin dificultades en el manejo de los niños o los equipos, supone una adecuada selección de los casos y preparación de los padres en las técnicas, así como apoyo profesional en el domicilio, condiciones indispensables que no pueden onnitirse en programas o experiencias similares ${ }^{12,13,24}$. La morbilidad durante el período de oxigenoterapia en domicilio fue relativamente escasa, menor a la citada en otros trabajos ${ }^{14,15}$; parece más bien relacionada con inmadurez que con la enfermedad pulmonar crónica misma. Las infecciones intercurrentes fueron excepcionales.

La oxigenoterapia en domicilio puede ser una 
opción terapéutica ventajosa en casos de displasia broncopulmonar bien seleccionados. Permite abreviar significativamente la hospitalización, mejorando la relación familiar y probablemente disminuyendo costos, pero exige condiciones familiares y de apoyo ambulatorio que no pueden ser omitidas.

\section{RESUMEN}

Con el objeto de evaluaz la factibilidad, venta. jas y complicaciones de la oxigenoterapia ambulatoria en el tratamiento de la displasia broncopulmonar (DBP) se estudiaron 8 casos de DBP grave dados de alta del servicio de neonatología de una clínica privada de Santiago. La edad gestacional era $27 \pm 2$ semanas, el peso al nacer $1.181 \pm 353$ $\mathrm{g}$, el alta se dio con oxemia estable con oxigeno en naricera y flujo inferior a $1,51 \cdot \mathrm{min}, \mathrm{PCO}_{2}$ menor de $50 \mathrm{mmHg}$ y peso ascendente. Antes del alta se adiestró a los padres en la administración del oxígeno y en técnicas básicas de reanimación. Se administró oxígeno en domicilio por 71 días en promedio (rango 7 a 339), con buena aceptación familiar, sin dificultades ni complicaciones y sólo dos rehospitalizaciones, anbas por bronconeumonía. El incremento ponderal se mantuvo entre los percentiles 3 y 50 . La oxigenoterapia en domicilio es una alternativa ventajosa en el tratamiento de la DBP.

(Palahras clave: displasia broncopulmonar, oxigenoterapia dorniciliaria.)

\section{REFERENCIAS}

1. Bancalari $E$., Gerhardt $T$, Bronchopulmonasy dy splasia. Pediatric Clinics of N.A. 1986; 33: 1-23.

2. Montin P., Vert P.: The management of bronchopulmonary dysplasia. Clinics in Perinatology 1987 ; 14: 531-549.

3. The HIFI Study Group: High frequency oscilatory ventilation compared with conventional mechanical ventilation in the treatment of respiratory failure in preterm infants. $N$ Engl J Med 1989; 320: $88-93$.

4. Bece J.P., Wllson J., Bernier L., Torres J.: Dysplasia broncopulmonar grave. Rev Chil Pediatr 1985; 56: $242-246$.

5. Bece J.P., Torres J., Sfeir J., Rodriguez G., Pefla $M$. , Vila $A$. .: Oxigenoterapia en domicilio en pacientes con displasia broncopulmonar. XIII Jornadas Chilenas de Pediatría. Libro de Resúmenes, p. 9.

6. Skérmeto $M$., Rizzardini $\boldsymbol{M}$.: Displasia broncopulmonar: Estudio clínico. XIII Jornadas Chilenas de Peóiatría, Libro de Resúmenes, p. 61.
7. Bancolari $E$., Abdenour G.E., Feller $R$., Gannon $J$.: Bronchopulmonary dysplasia: Clinical presentation. J Pediatr 1979; 95: 819-823.

8. National Center for Health Statistics. NCHS Growth Charts 1976; Monthly Vital Statistics Report. Vol. 25, No 3. Supp (HRA) 76-1120. Health Resources Administration. Rockville, Maryland, June 1976. Yellow Springs, Ohio.

9. Avery M,E., Tooley W., Keller J.: Is chronic lung disease in low birthweight infarts preventable? A survey in eight centers. Pediatrics 1987; 79: 2630.

10. Lawson E.E.: Vitamin A. Therapy for prevention of chronic lung disease in infants. J Pediatr 1987; 111: 247-148.

11. Shenai J.P., Kennedy K.A., Chytil F.: Clinical trial of Vitamin $A$ supplementation in infants susceptible to bronchopulmonary dysplasia. J Pediatr 1987; 111: 269-277.

12. Pinney M.A, Cotion E.K.: Home management of bronchopulmonary dysplasia. Pediatrics 1976; 58 : 856-859.

13. Camphell A.M., Zaffin Y., Groenveld M., Bryan $M . H .+$ Low flow oxygen therapy in infants. Arch Dis Child 1983 ; 58 : 795-798.

14. Doily D.K.: Home oxigen therapy for infants with bronchopulmonary dysplasia, Perínatology-Neonatology $1987 ; 11: 26-35$.

15. Samuels M.P. Warber J.O.* Bronchopulmonary dysplasia: The outcome. Arch .Dis Child 1987; 62: 1099-1101.

16. Groothuis J.R., Rosenberg A.A.: Home oxigen promotes weight gain in infants with bronchopulmonary dysplasia. Am J Dis Child 1987; 141: 992-995.

17. Philip A.G.S., Peabody J.L., Lucey J.F.: Transcutaneous $\mathrm{PO}_{2}$ monjtoring in the management of bronchopulmonary dysplasía. Pediatrics 1978; 61: 655-657.

18. Yeh T.F., Mc Clenan D.A., Aloyi O.A., Pildes R.S.: Metabolic rate and energy balance in infants with bronchopulmonary dysplasia, J Pediatr 1989; 114: 448-151.

19. KoO L.C., Durand D.J., Nickerson B.G.: Improving pulmonary function does not decrease oxygen consumption in infants with bronchopulmonary dy splasia. J Pediatr $1988 ; 112: 616-621$.

20. Kurtmer S.J., Garg M, Bautista D.B.: Growth failure in bronchopulmonary dy plasia: Elevated metabolic rates and pulmonary mechanics. $\mathfrak{J}$ Pediatt 1988; $112: 73-80$.

21. Goodman G., Perkin R.M., Anas R.G.: Pulmonary hypertension in infants with bronchopulmonary dysplasia. J Pediatt 1988; 112:67-72.

22. Werthommer J., Brown E.R., Neff R,X., Toeusch $H . W$. : Sudden infant death syndrome in infants with bronchopulmonary dy splasia. Pediatrics 1982; 69: $301-304$.

23. Donn $S$ : Cost effectiveness of home management of bronchopulmonary dysplasia. Pediatrics 1982; 70: $330-331$.

24. Sewell E.M., Holsclaw D., Schidlow D., Mc Geady $S$. Berger $B$.: The use of oxygen for children in their homes. Pediatric Pulmonology 1986; 2: 72 73. 